\title{
Quantification and Compensation of the Influence of Pulse-Transients on Symmetry-Based Recoupling Sequences
}

Johannes J. Wittmann ${ }^{\mathrm{a}}$, Valerie Mertens ${ }^{\mathrm{a}}$, Kazuyuki Takeda ${ }^{\mathrm{b}}$, Beat H. Meier ${ }^{\mathrm{a}^{*}}$, Matthias Ernst $\mathrm{t}^{\mathrm{a}^{*}}$

aPhysical Chemistry, ETH Zurich, Vladimir-Prelog-Weg 2, 8093 Zurich, Switzerland

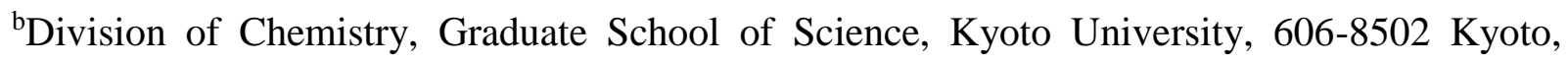
Japan

Corresponding Authors:

Beat H. Meier, Matthias Ernst

Physical Chemistry, ETH Zürich, Vladimir-Prelog-Weg 2, 8093 Zürich, Switzerland

E-mail: beme@ethz.ch,maer@ethz.ch

Phone: +41 4463244 01, +41 446324366 


\begin{abstract}
Deviations of amplitude and phase of radio-frequency pulses from the desired values, can have a severe impact on the performance of multiple-pulse sequences in NMR spectroscopy. A particular problem are pulse transients that appear every time there is a discontinuity in amplitude or phase. Based on a Floquet description using pulses with arbitrarily shaped amplitudes and phases we present a systematic study of the influence of pulse transients on symmetry-based pulse sequences in solid-state NMR under magic-angle spinning. This treatment explains the dependence of the experimentally observed transfer efficiency on the details of experimental setups. In addition, three approaches are compared which have the aim to re-establish highly efficient recoupling. We demonstrate that the application of transient-compensated pulses as basic elements of symmetry-based sequences leads to a significantly improved robustness of the experiments with respect to variations in the experimental setup.
\end{abstract}

\title{
Keywords
}

NMR spectroscopy, pulse transients, linear-response theory, symmetry-based recoupling 


\section{Introduction}

The design of NMR pulse sequences is usually based on the assumption that amplitude and phase of the radio-frequency (rf) field acting on the spins are identical to the amplitude and phase that are programmed on the spectrometer and also used in analytical calculations, numerical simulations or schematic representations of NMR pulse sequences. For example, hard pulses are usually represented by ideal rectangles, i.e. the amplitude of the pulse is turned on and off instantaneously and the phase is constant throughout the pulse. Experimentally, however, significant deviations of the actual $B_{1}$ fields, which act on the nuclear spins inside the tuned and matched resonance circuit, are observed [1,2] (Figure 1A and B). Such deviations of the phase and amplitude of the $B_{1}$ field from the ideal pulse shape are most prominent when amplitude or phase of rf pulses are changed instantaneously and are then usually referred to as pulse transients. Several analytical models which describe the origin of pulse transients in more or less complex model circuits can be found in literature [1,3-6]. Additional non-idealities include variations on a typically longer timescale caused by changes in the tuned circuit, e.g. by heating effects, or nonidealities of the power amplifiers, e.g. droop.

The altered spin trajectory due to the pulse transients is known to affect the performance of multiple-pulse sequences for line narrowing in static samples and under slow MAS $[7,8]$. Special tune-up sequences [9-12] have been designed to minimize the phase-transient effects by optimizing the tuning and matching of the amplifier and the probe such that in second-order average Hamiltonian theory [7] (AHT) the effective rotation axes of the pulses are close to the ideal ones. Such tune-up sequences had been used extensively while tube amplifiers with tunable output impedance were used in NMR spectrometers. At present, these tune-up schemes are rarely used, as linear solid-state amplifiers have become the standard equipment in commercial NMR spectrometers and do not allow adjustment of the output impedance. In principle, a detuning of 
the probe or an adjustment of the cable length between preamp and probe also allows a minimization of the quadrature pulse transients (Figure S1). The first approach, even though it can be accomplished easily, has the disadvantage, that it leads to decreased sensitivity and increased pulse power. An adjustment of the cables is time-consuming, especially, if the elimination of phase-dispersive peaks in nutation spectra is used as an optimization criterion. Alternatively, including a tunable LC circuit has been used in experimental setups to minimize anti-symmetric phase transients [12].

The impact of pulse transients on the performance of pulse sequences used for high-resolution solid-state NMR under MAS has attracted only little attention in recent years, despite the fact that they can lead to significant variations in the results depending on the experimental setup. The effects of pulse transients have been studied in detail in homonuclear-decoupling sequences based on phase-modulated Lee-Goldburg [4,13] irradiation in order to obtain a rotation of the magnetization around the z-axis. The influence of phase transients on the performance of symmetry-based recoupling sequences [14,15] was analyzed using simulations [6] and a modification of the symmetry-based double-quantum recoupling sequence POST-C7 [16] was proposed using a phase-tuned POST element $(\pi / 2)_{\xi}(2 \pi)_{\pi}(3 \pi / 2)_{0}$, where the $\pi / 2$ pulse is phase shifted [5]. By experimental optimization of the phase $\xi$, double-quantum recoupling efficiencies increased towards the theoretical maximum. All experimental approaches to compensate the effects of phase transients suggested so far require optimization of the NMR signal on the spectrometer, which is difficult to achieve for samples with low signal-to-noise ratio. Furthermore, the optimization is specific to the given experimental setup and requires an adjustment every time the experimental conditions change.

In a different approach, the compensation of pulse transients is implemented in the pulse programmer. By measuring the impulse response of the tuned circuit using a small pick-up coil 
close to the NMR rf coil, the transfer function of the resonance circuit can be determined based on linear-response theory. This allows the calculation of the required input pulse shape to generate the desired pulse shape in the coil of the probe [17-19]. Such transient-compensated pulses have been used in nutation experiments and for minimization of the receiver dead-time as well as to compensate for limited resonator bandwidth in EPR spectroscopy [19-22]. Recently, we have shown that transient-compensated pulses can be used in symmetry-based recoupling sequences to obtain consistent and reproducible results to a large degree independent of the experimental setup [23]. This approach requires some modifications of the basic element used in the symmetry-based sequences. However, the "digital tune up" of the spectrometer can be done independent of the NMR signal since the correction of the input signal depends only on the characteristics of the resonance circuit.

In this publication, we extend our earlier communication [23] and present a systematic analysis of the influence of pulse transients on symmetry-based sequences using the double-quantum recoupling sequence POST-C7 as an example. The experimental findings are rationalized using an extended theoretical description of symmetry-based C-sequences based on triple-mode Floquet theory. Furthermore, we demonstrate how the characteristics of the resonance circuit can be determined experimentally to be used for the calculation of transient-compensated pulses. With the results from our Floquet analysis we derive modifications of the commonly used POST element, which are necessary, if transient-compensated pulses are used in the basic elements. We show that high double-quantum scaling factors and a simultaneous suppression of unwanted interactions, like the chemical shift, can be achieved. We demonstrate that the application of the transient-compensated sequences leads to a gain in efficiency and a high reproducibility between different experimental setups. Furthermore, we show, that the concepts developed for C sequences can also improve the performance of R-type family of pulse sequences. 

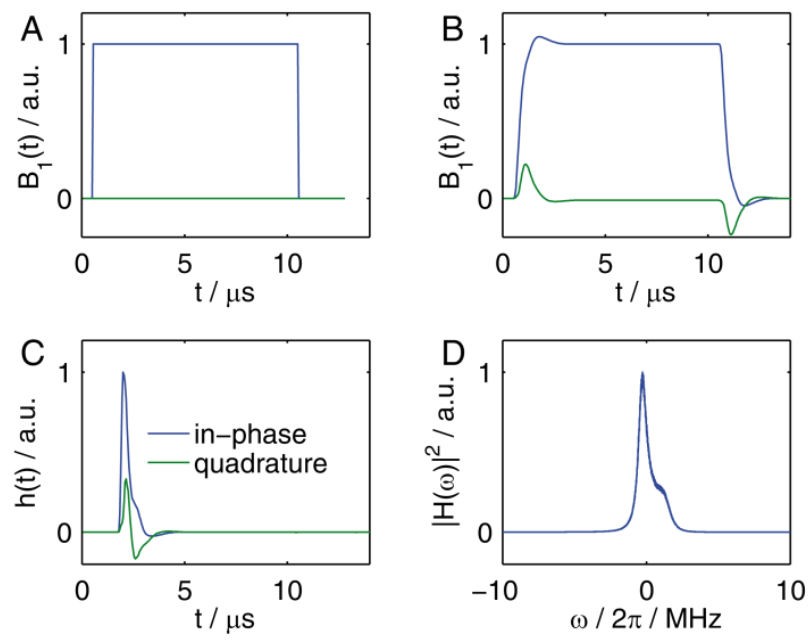

Figure 1: A) Ideal pulse envelope for a rectangular excitation and B) corresponding experimental probe response illustrating the exponential build-up and decay of the in-phase component of the rf field (blue) as well as $90^{\circ}$ phase-shifted components (green) in the beginning and the end of the pulse. C) Corresponding impulse-response function $h(t)$ determined using an MLS-12 sequence and D) absolute square of Fourier transform of $h(t)$.

\section{Transient-Compensated Pulses}

\subsection{Linear-Response Theory and Determination of the Impulse-Response Function}

Transient-compensated rf-pulses can be obtained using linear response theory [18-20]. In this framework, the time-domain response $y(t)$ of a system is given by the convolution of the input signal $x(t)$ with the impulse-response function $h(t)$, which contains all the information about the system:

$$
\begin{aligned}
y(t) & =x(t) \otimes h(t) \\
& =\int_{-\infty}^{\infty} x(t) h(t-\tau) \mathrm{d} \tau \\
& =\mathcal{F}^{-1}\{\mathcal{F}\{x(t)\} \mathcal{F}\{h(t)\}\} \\
& =\mathcal{F}^{-1}\{X(\omega) H(\omega)\}
\end{aligned}
$$

In the frequency domain, the convolution is given by the product of the Fourier transforms of $x(t)$ and $h(t)$. The Fourier transforms of the individual signals are denoted by the corresponding 
capital letters. The system characterized by $h(t)$ (an example for $h(t)$ is shown in Figure 1C) includes all the elements of the rf circuit of the NMR spectrometer, such as probe, amplifiers, filters, cables, etc. Also the influence of the exact experimental conditions (e.g., tuning, matching as well as the deviation of the carrier frequency of the pulses from the resonance frequency of the circuit) are contained in this function. Even though some of the components, like the high-power amplifiers, can exhibit non-linear features, linear behavior is assumed in first-order approximation.

Among the several ways of characterizing the impulse-response function of the system [24], we chose maximum-length sequences (MLS) [25], which have already been used for system identification in EPR spectroscopy [20]. These cyclic pseudo-random noise sequences have the property that their auto-correlation function approximates a delta-function

$$
\operatorname{MLS}(t) \otimes M L S(t)=\delta(t)
$$

Thus, a cyclic cross-correlation of the MLS- $N$ with the probe response to a $\operatorname{MLS}-N$ input signal will yield the impulse-response function [25]

$$
\operatorname{MLS}(t) \otimes y^{\mathrm{MLS}}(t)=\operatorname{MLS}(t) \otimes(M L S(t) \otimes h(t))=h(t)
$$

In Eq. (6) we used the relation given in Eq. (5) to calculate the probes response $y^{\mathrm{MLS}}(t)$. The result follows from the associative properties of the convolution. A MLS of given order $N$ consist of $2^{N}-1$ bits. The value of each bit can be calculated from an initial seed using a linear feedback shift register and can only take the values +1 or -1 . With a time step $\Delta t$ the total duration of a cycle is given by $\tau_{\mathrm{MLS}}=\left(2^{N}-1\right) \Delta t$. For meaningful results $\Delta t$ needs to be short enough such that the excitation bandwidth of the pseudo-random noise sequence is larger than the resonator bandwidth. On the other hand, $h(t)$ needs to have decayed during the time $\tau_{\mathrm{MLS}}$ of a 
single cycle. It has to be emphasized that for reasonable results the cyclic properties of the maximum-length sequences have to be maintained. Therefore, the resonator has to be excited by a series of at least two MLS cycles and only the response towards the second cycle is used for the calculation of $h(t)$, i.e., the system is given time to equilibrate during the first MLS [25]. Note, that for typical solid-state NMR probes a time step of $\Delta t=50 \mathrm{~ns}$ is sufficient requiring a MLS with a minimum order $N_{\min } \approx 7$.

Knowing the impulse-response function, a deconvolution by division in the frequency domain allows the calculation of the input waveform $x(t)$ that results in the target waveform $y(t)$.

$$
x(t)=\mathcal{F}^{-1}\left\{\frac{Y(\omega) H^{*}(\omega)}{|H(\omega)|^{2}} W(\omega)\right\} .
$$

The asterisk denotes the complex conjugate. Even though MLS allows the determination of $h(t)$ with a high signal-to-noise ratio, the noise on the experimental data can interfere with the deconvolution procedure. Especially at higher frequency, where $|H(\omega)|^{2}$ has decayed to values close to zero (Figure 1D), small variations of the actual values due to noise can lead to a overamplification of high-frequency components in the compensated shapes. Therefore, we applied a Tukey window [26] $W(\omega)$ before inverse Fourier transform.

In practice, it is only possible to generate pulse waveforms without discontinuities. Accordingly, we chose to implement pulses of length $\tau_{\mathrm{p}}$ with well-defined amplitude-shaped edges with a rise or fall time $\tau_{\text {edge }}$.

$$
\omega_{1}(t)=\omega_{1}^{\max } \cdot \begin{cases}\sin ^{2}\left(\frac{\pi t}{2 \tau_{\text {edge }}}\right) & 0<t \leq \tau_{\text {edge }} \\ 1 & \tau_{\text {edge }}<t<\tau_{\mathrm{p}}-\tau_{\text {edge }} \\ \sin ^{2}\left(\frac{\pi\left(\tau_{\mathrm{p}}-t\right)}{2 \tau_{\text {edge }}}\right) & \tau_{\mathrm{p}}-\tau_{\text {edge }} \leq t<\tau_{\mathrm{p}}\end{cases}
$$


Unless very high peak rf fields are acceptable, the rise and fall times have to be in the order of the characteristic time constant of the resonance circuit (Figure 2). For the probe and spectrometer used in our experiments, feasible rise times were on the order of a few hundred nanoseconds. If those amplitude-shape pulses are used inside the symmetry-based sequences the sudden phaseswitching required between different pulses takes place at the time, where the amplitude is zero, such that potential distortions due to this discontinuity have little influence on the spin dynamics. All (composite) pulses with amplitude-shaped edges will be marked with a lower-case letter "a" to emphasize the shaped edges.

\subsection{Quality of Experimental Transient Compensation}

Figure 1B shows the response of the NMR probe we used for our studies to a rectangular excitation (Figure 1A). The typical features due to the transient behavior of the tuned resonator namely a delayed buildup and overshooting of the in-phase rf-field as well as the appearance of quadrature components are clearly visible. The corresponding impulse-response function is shown in Figure 1C. In our case $h(t)$ was determined using a MLS-12 sequence. With the length per bit set to $\Delta t=50 \mathrm{~ns}$, the total length of the sequence is $204.75 \mu \mathrm{s}$. An example of transientcompensated shaped pulses and the corresponding probe responses are shown in Figure 2. Such pulses require an in-phase component that rises faster and overshoots compared to the desired pulse shape. For short values of $\tau_{\text {edge }}$ the peak rf fields can exceed the plateau values by several times (Figure 2A). The out-of-phase components of the compensated pulse at the rising edge have the opposite sign compared to the ones observed for the uncompensated pulse (Figure 1B),

consequently counteracting the formation of quadrature transients. Similar observations hold for the falling edge. The obtained probe responses are in good agreement with the desired target 
shape, which are pulses with sin-shaped edges. Only for the shortest transition time (250 ns, Figure 2A), small deviations are observed at the leading edge of the pulse. These are most likely caused by the high rf-field amplitude in combination with non-linearity of the amplifier. Nevertheless, the measured responses for transition times larger than $400 \mathrm{~ns}$ indicate, that transient-compensated pulses generate a well-defined amplitude-modulated $B_{1}$ field in the coil with only small deviations (RMSD $<2 \%$ ) from the intended pulse shape.
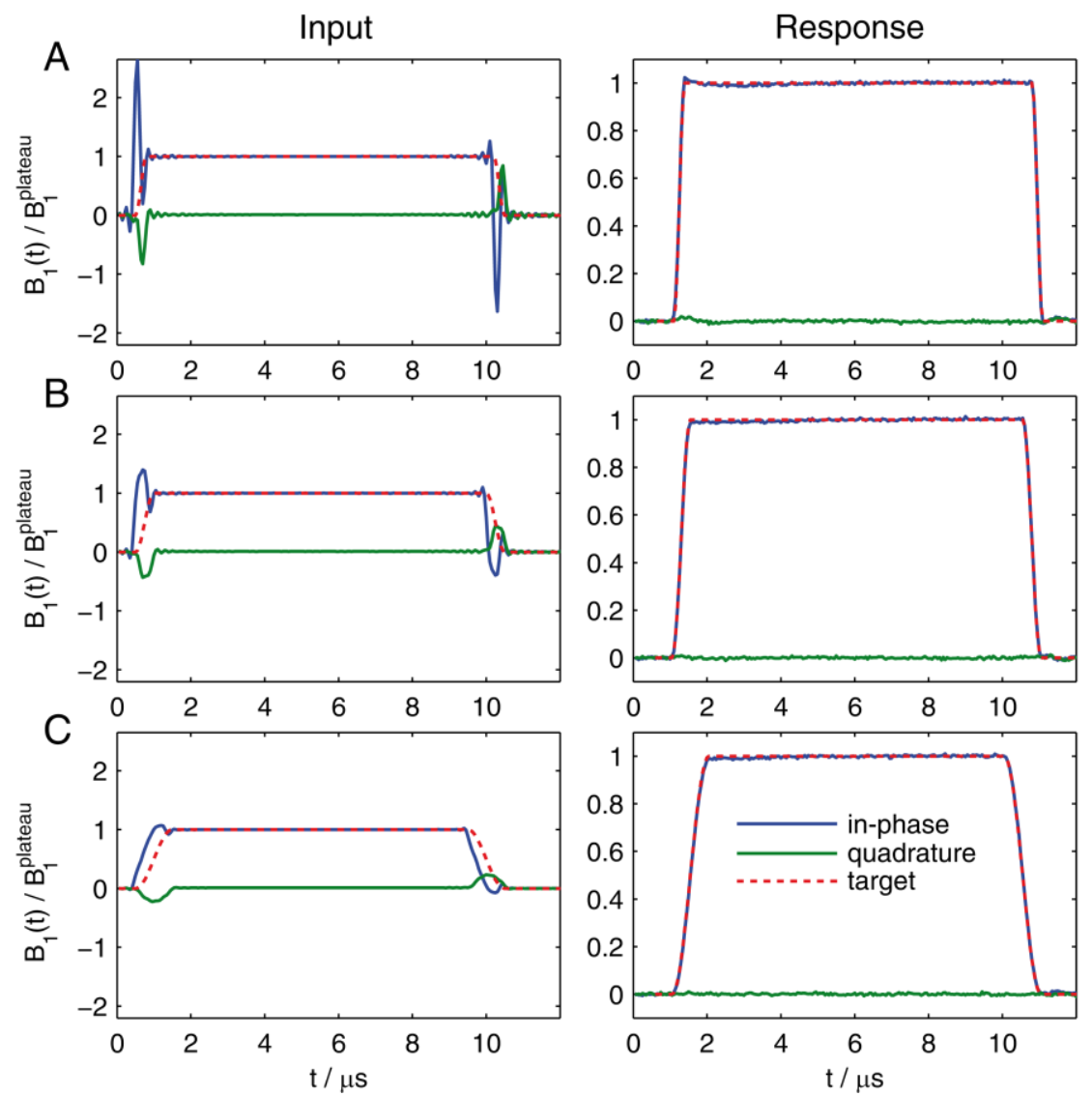

Figure 2: Compensated input shapes and corresponding responses of a commercial $1.9 \mathrm{~mm}$ Bruker double-resonance probe (resonance frequency $75 \mathrm{MHz}$ ) as a function of the transition time of the target shape: A) $250 \mathrm{~ns} \mathrm{~B}) 500 \mathrm{~ns}$ C) $1000 \mathrm{~ns}$. Increasingly higher maximum values of the in-phase component of the compensated input shape are required to compensate for smaller values of $\tau_{\text {edge. }}$ The quality of the compensation is good and independent of the transition time. Only for transition times smaller than $300 \mathrm{~ns}$ small deviations are observed in the compensated response (A) caused by non-linear features of the amplifier. 


\section{$3 \quad$ Pulse Transients and Symmetry-Based Recoupling}

\subsection{Theoretical Description of Symmetry-Based Sequences}

Following the rules for symmetry-based $\mathrm{CN}_{n}^{v}$ sequences [14,15], $N$ elements (C) with a flip angle of an integer multiple of $2 \pi$ are fit into $n$ rotor periods. With each repetition of the basic element the phase is incremented by $2 \pi v / N$. In principle, any composite pulse corresponding to a unity propagator can be used as a basic $\mathrm{C}$ element. The most popular implementation of the $\mathrm{C}$ elements are $(2 \pi)_{\varphi}(2 \pi)_{\varphi+\pi}$ and the POST element $(\pi / 2)_{\varphi}(2 \pi)_{\varphi+\pi}(3 \pi / 2)_{\varphi}$ which provides better offset compensation [14,27]. In the case of symmetry-based $\mathrm{R} N_{n}^{v}$ sequences, $N / 2$ elements $\left(R_{\phi} R_{-\phi}\right)$ where each $R$ element has a flip angle of an integer multiple of $\pi$ are fit into $n$ rotor periods. Instead of a simple $\pi$ pulse also a composite $(\pi / 2)_{\varphi}(3 \pi / 2)_{\varphi+\pi}$ pulse is often used. The phase $\phi$ for each of the $R$ elements is given by $\pi v / N$. For both types of sequences, a proper selection of the symmetry coefficients $N, n$ and $v$ allows a selective recoupling of specific interactions based on their symmetries under rotations in real and spin space. A schematic representation of the doublequantum recoupling sequences POST-C $7 \frac{1}{2}$ and $\mathrm{R} 14_{2}^{6}$, which are used as examples in this paper, are shown in Figure 3. 

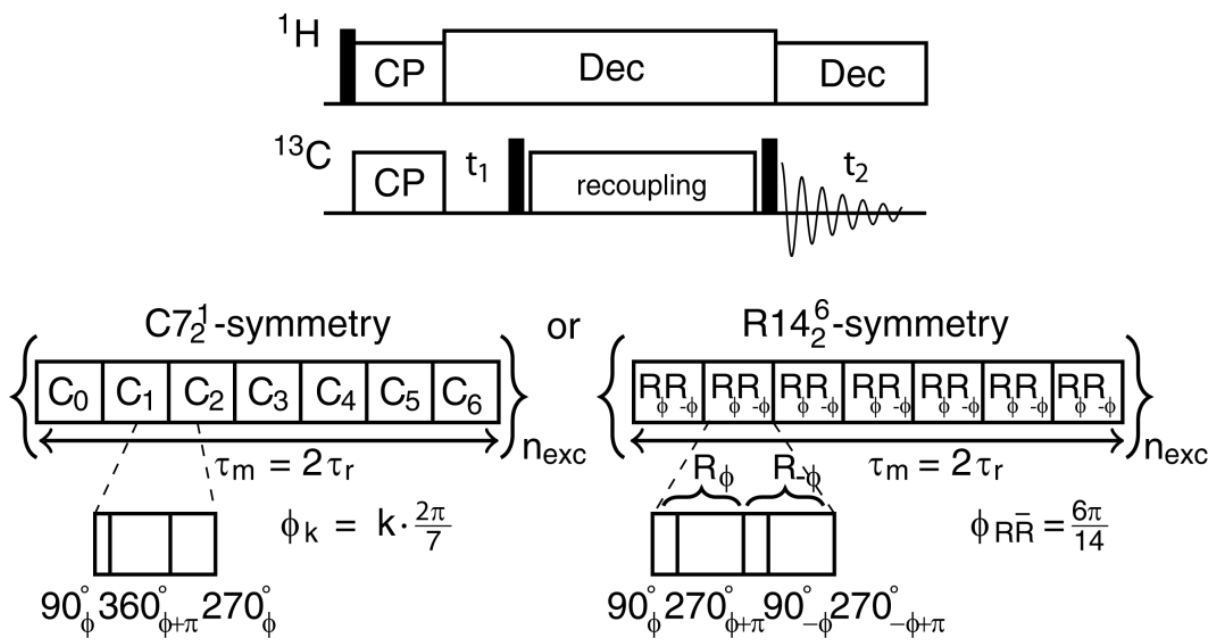

Figure 3: Schematic representation of a 2D correlation experiment using the symmetry-based DQ recoupling sequences for DQ recoupling. The composite pulse POST is used as C-element in the $\mathrm{C7} \underset{\mathbf{2}}{\mathbf{1}}$ symmetry. For R14${ }_{2}^{\mathbf{6}}$ a $\mathbf{9 0}_{\boldsymbol{\phi}} \mathbf{2 7 0}_{\boldsymbol{\phi}+\boldsymbol{\pi}}$ pulse is used as $\boldsymbol{R}_{\boldsymbol{\phi}}$ element.

The theoretical description of symmetry-based pulse sequences can be done using either averageHamiltonian theory [14], or bi-modal Floquet theory [28,29] where the two relevant frequencies are the modulation frequency of the pulse sequence, $\omega_{\mathrm{m}}=2 \pi / \tau_{\mathrm{m}}$, and the MAS frequency, $\omega_{\mathrm{r}}$. In such a description, it is assumed that the basic element is an identity propagator for the C-type sequences and an ideal inversion propagator for the R-type sequences. In a more generalized description, this restriction is removed and a tri-modal Floquet description including also the effective field of the sequence, $\omega_{\text {eff, }}$ is required similar to the description of asynchronous $\mathrm{C}$ sequences [30].

For our discussion, we assume a homonuclear dipolar-coupled two-spin system $(p=1,2)$ including the chemical-shift interaction during a symmetry-based sequence. In spherical-tensor notation the rotating-frame Hamiltonian is given by

$$
\mathcal{H}(t)=\sum_{p} \omega_{p}(t) T_{1,0}^{(p)}+\omega_{1,2}(t) T_{2,0}^{(1,2)}+\mathcal{H}_{\mathrm{rf}}(t)
$$


with

$$
\mathcal{H}_{\mathrm{rf}}(t)=\omega_{1}(t) \sum_{p}\left(\cos (\phi(t)) S_{p x}+\sin (\phi(t)) S_{p y}\right)
$$

Here, $\omega_{p}$ denotes the chemical shift of $\operatorname{spin} p, \omega_{1,2}$ the dipolar interaction. $\omega_{1}$ and $\phi$ are amplitude and phase of the applied rf field, respectively. The spherical-tensor operators are defined as $T_{1,0}^{(p)}=S_{p z}$ and $T_{2,0}^{(1,2)}=-\frac{1}{\sqrt{6}}\left[3 S_{1 z} S_{2 z}-\vec{S}_{1} \cdot \vec{S}_{2}\right]$. In the most general case, where an explicit time dependence of rf-field amplitude and phase is taken into account, the interaction-frame transformation of the spin-system Hamiltonian by the radio-frequency Hamiltonian is described by

$$
\widehat{U}(t)=\widehat{T} \exp \left(-i \int_{0}^{t} \mathcal{H}_{\mathrm{rf}}\left(t^{\prime}\right) d t^{\prime}\right)
$$

Here, $\hat{T}$ is the Dyson time-ordering operator [31]. Any periodic pulse sequence can be characterized by two frequencies, the modulation frequency, $\omega_{\mathrm{m}}$, which depends on the cycle time of the sequence, and the effective field of the pulse sequence, $\omega_{\text {eff. }}$ For a symmetry-based pulse sequence with a standard $\mathrm{C}$ element and ideal rectangular pulses, $\widehat{U}(\mathrm{C})=\hat{1}$ and the effective field $\omega_{\text {eff }}$ over the complete sequence vanishes. For an R-type sequence with ideal $\pi$ pulses one also finds $\omega_{\text {eff }}=0$ over a full cycle, i.e., $\widehat{U}\left(\mathrm{R} N_{n}^{v}\right)=\hat{1}$. In case of non-ideal pulses, due to phase transients or amplitude-shaped pulses, this assumption can be violated $(\widehat{U}(\mathrm{C}) \neq \hat{1}$ and $\left.\widehat{U}\left(R N_{n}^{v}\right) \neq \hat{1}\right)$ and we have to include all three frequencies into the theoretical description. In the general case with $\omega_{\text {eff }} \neq 0$, the interaction-frame Hamiltonian $\widetilde{\mathcal{H}}$ can then be written as a Fourier series

$$
\tilde{\mathcal{H}}(t)=\sum_{n=-2}^{2} \sum_{k=-\infty}^{\infty} \sum_{\ell=-2}^{2} \widetilde{\mathcal{H}}^{(n, k, \ell)} e^{i n \omega_{\mathrm{r}} t} e^{i k \omega_{\mathrm{m}} t} e^{i \ell \omega_{\mathrm{eff}} t}
$$


with the Fourier coefficients $\widetilde{\mathcal{H}}^{(n, k, \ell)}$ given by:

$$
\begin{gathered}
\tilde{\mathcal{H}}^{(0, k, \ell)}=\sum_{p=1}^{2} \omega_{p}^{(0)} \sum_{s=-1}^{1} a_{1, s}^{(k, \ell)} T_{1, s}^{(p)} \\
\widetilde{\mathcal{H}}^{(n, k, \ell)}=\omega_{1,2}^{(n)} \sum_{s=-2}^{2} a_{2, s}^{(k, \ell)} T_{2, s}^{(1,2)}+\sum_{p=1}^{2} \omega_{p}^{(n)} \sum_{s=-1}^{1} a_{1, s}^{(k, \ell)} T_{1, s}^{(p)}
\end{gathered}
$$

The scaling factors $a_{r, s}^{(k, \ell)}$ are the Fourier coefficients of the interaction-frame transformation of the $T_{r, 0}$ spin-tensor operators and can for ideal rectangular pulses be calculated analytically [29]. In the case of non-ideal or amplitude-modulated pulses, however, they have to be determined by a numerical Fourier transformation of the interaction-frame trajectory. Details are given in section 5. The first-order effective Hamiltonian $\overline{\mathcal{H}}^{(1)}$ at the tri-modal resonance condition

$$
n_{0} \omega_{\mathrm{r}}+k_{0} \omega_{\mathrm{m}}+\ell_{0} \omega_{\mathrm{eff}}=0
$$

is given by triple-mode Floquet theory:

$$
\overline{\mathcal{H}}^{(1)}=\widetilde{\mathcal{H}}^{(0,0,0)}+\sum_{n_{0}, k_{0}, \ell_{0}} \widetilde{\mathcal{H}}^{\left(n_{0}, k_{0}, \ell_{0}\right)}
$$

Here, the summation includes all values of $n_{0}, k_{0}$ and $\ell_{0}$ for which the resonance condition of Eq. (15) is fulfilled. In general, there are no restrictions on the possible values of $n_{0}, k_{0}$ and $\ell_{0}$ and the strength of the recoupling condition is determined by the magnitude of the scaling factors $a_{r, s}^{(k, \ell)}$. If we assume that the effective field is small compared to the spinning frequency, i.e. if we 
stay close to the resonance condition of the ideal symmetry-based sequence, we can simplify the discussion. For ideal pulses, i.e., $\omega_{\mathrm{eff}}=0$, the bimodal resonance condition is given by

$$
n_{0} \omega_{\mathrm{r}}+k_{0} \omega_{\mathrm{m}}=0
$$

For the $\mathrm{C} 7 \frac{1}{2}$ sequence, this leads to the condition $-n n_{0}=k_{0}(n \in \mathbb{N})$ which has to be fulfilled, i.e., $n_{0}= \pm 1$ and $k_{0}=\mp 2$. One can show that under $\mathrm{C}$ symmetry the coefficients $a_{r, s}^{(k, 0)}$ are non-zero only for $k=z N-s v$ where $z$ is an integer[29]. Therefore, only the term $a_{2, \pm 2}^{(\mp 2,0)}$ will be selected and the double-quantum terms $\omega_{1,2}^{( \pm 1)} a_{2, \pm 2}^{(\mp 2,0)} T_{2, \pm 2}^{(1,2)}$ will be recoupled. For small values of $\omega_{\text {eff }}$ the axis of the additional nutation for a $\mathrm{C}$ sequence is close to the $\mathrm{z}$ axis and the coefficients $a_{r, s}^{(k, \ell)}$ are non-zero only for $k=z N-s v$ and $\ell=-s$. In the case of arbitrarily-shaped pulses, the timeindependent double-quantum terms are, therefore, contained in $\widetilde{\mathcal{H}}^{( \pm 1, \mp 2, \mp 2)}$ and scale with $a_{2, \pm 2}^{(\mp 2, \mp 2)}$

$$
\widetilde{\mathcal{H}}^{( \pm 1, \mp 2, \pm 2)}=\omega_{1,2}^{( \pm 1)} a_{2, \pm 2}^{(\mp 2, \mp 2)} T_{2, \pm 2}^{(1,2)}
$$

Therefore, we observe still a double-quantum recoupling but the resonance conditions are altered compared to the sequence with ideal pulses. In first-order approximation, the most important error terms originate from the non-resonant terms

$$
\widetilde{\mathcal{H}}^{(0,0,0)}=\sum_{p} \omega_{p}^{(0)} \sum_{s=-1}^{+1} a_{1, s}^{(0,0)} T_{1, s}^{(p)}
$$

which are proportional to the isotropic chemical shift. They can lead to an effective field along an arbitrary axis in the interaction frame. However, it is often sufficient to consider only the coefficient $a_{1,0}^{(0,0)}$ as chemical-shift scaling factor, as the transverse components are averaged over 
one symmetry cycle of the $\mathrm{C}$ sequence. In order to obtain good double-quantum recoupling, the scaling factor for the desired resonant term $\left(a_{2, \pm 2}^{(\mp 2, \mp 2)}\right)$ has to be as large as possible while simultaneously the scaling factor for the unwanted isotropic chemical shift $\left(a_{1,0}^{(0,0)}\right)$ should be

minimized. More details how the scaling factors $a_{r, s}^{(k, \ell)}$ are calculated can be found in the Materials and Methods section. Similar arguments can be made for the R-type sequences [28] .

\subsection{Influence of Pulse Transients on POST-C7 Double-Quantum Recoupling}

As mentioned above, the exact shape of the pulse transients and, thus, the value of the effective field, is dependent on the actual experimental setup and can, for example, be influenced in a reproducible way by changing the length of a cable in the circuit, e.g. between preamplifier and probe. These changes are also used as a proxy for other changes encountered in the experiments including different probes, probe tuning, dielectric properties of the sample, impedance differences between amplifiers and preamplifiers, between different $\mathrm{rf}$ channels or spectrometers. In order to illustrate the effect such differences in the experimental setup can have on the recoupling behavior of symmetry-based sequences, the DQ transfer between the two selectively labeled carbonyl groups in di-ammonium phthalate was measured as a function of the rf-field amplitude for three cables of different length. Due to the relatively small dipolar coupling of $585 \mathrm{~Hz}$ deviations from the resonance condition and potential error terms, which might be introduced by the pulse transients, are already expected to have a strong influence on the recoupling performance. Recoupling even smaller couplings becomes increasingly more critical. The experimental results in Figure 4 (first row) clearly show significant differences of the DQ transfer as a function of the rf-field strength between the three experimental setups. Both, the maximum polarization-transfer efficiency as well as the $\mathrm{rf}$ field required for optimum transfer 
vary drastically between the different setups (first row of Figure 4). In case of setup A (125 cm cable, first column) two optima with different efficiencies are found in the measured range of rf fields. The setup B (cable of length $155 \mathrm{~cm}$ ) shows a single reduced polarization-transfer maximum at an rf field of about $76 \mathrm{kHz}$. In contrast, no significant DQ transfer is observed in the field-range investigated in case of setup C (cable of length $170 \mathrm{~cm}$ ). For an excitation with a rectangular pulse shape, the corresponding $B_{1}$ fields inside the coil are shown in the second row of Figure 4. It is interesting to see that setup $\mathrm{C}$, which has the smallest quadrature transients, shows the worst performance in terms of polarization-transfer efficiency. This behavior can be understood from the magnitude of the effective field of the C7 sequence, which was calculated for the experimental phase-transient profiles. Good polarization transfer is only observed whenever the effective field of the $\mathrm{C} 7$ sequence is zero and the classic bimodal resonance condition between $\omega_{m}$ and $\omega_{r}$ is fulfilled. In fact, these are the only conditions, where the calculated bimodal DQ scaling factors $a_{2, \pm 2}^{(\mp 2,0)}$, i.e. where only the Fourier coefficients at the synchronous resonance condition (Eq. (17)) are considered, are non-zero (Figure 4, row 4). However, the chemical-shift scaling factors $a_{1,0}^{(0,0)}$ (Figure 4, row 5) at the corresponding rf fields are in general not zero, indicating a reduced stability against chemical-shift offsets. In the sample used here, the chemical-shift difference between the resonances is small (5.9 ppm) and the most important unwanted terms originate from second-order CSA-CSA cross terms (last row of Figure 4) in the Hamiltonian, which have been calculated according to Tan et al. [30]. Their magnitude can explain the observed transfer efficiencies, as the DQ transfer is attenuated more strongly the larger these error terms become. Note, that the discontinuities in the CSA scaling factors at the conditions of $\omega_{\text {eff }}=0$ are due to the fact, that the suppression of the CSA-CSA cross terms is better for implementations of POST-C7 where the triple-frequency resonance condition with the additional effective field is matched [30]. 
In summary, transfer is only observed at rf-field amplitudes where the effective field of the C7 sequence originating from the pulse transients is zero due to "miss-setting" of the rf field and the classic resonance condition without the effective field is matched. The position of good polarization transfer, however, can be significantly shifted away from the theoretical rf-field amplitude. Experimental optimization of the rf-field amplitude for each experimental setup often leads to reasonable polarization transfer. However, the scaling factors of potential error terms are no longer minimal at these optimized rf fields leading to further degradation of the experimental performance. 

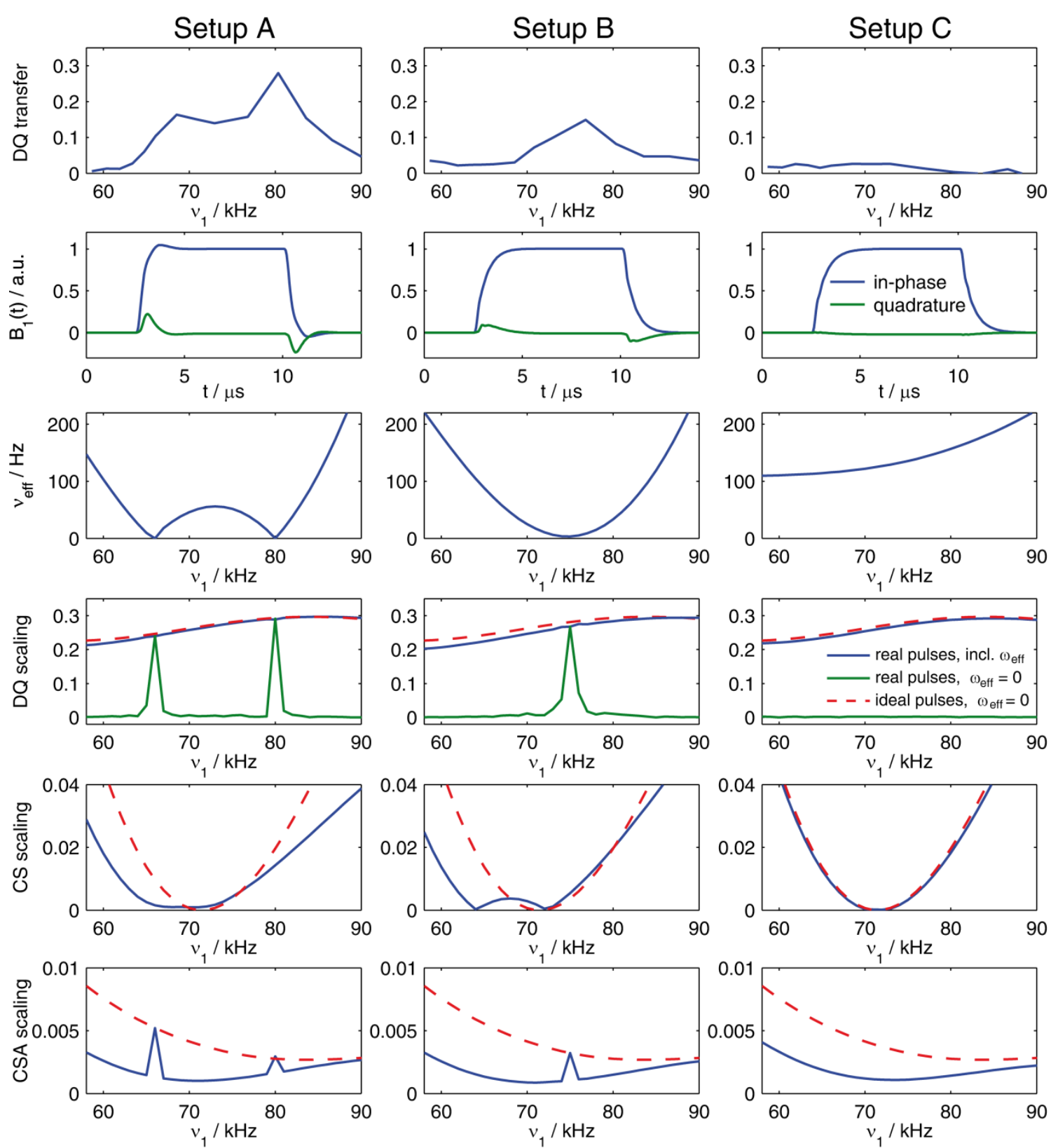

Figure 4: Influence of pulse transients on performance of POST-C7 double-quantum recoupling. In order to mimic differences in the experimental setup DQ transfer at a mixing time of $6.3 \mathrm{~ms}$ (first row) was recorded at an external B-field of $7.1 \mathrm{~T}$ for three cables of length A) $125 \mathrm{~cm}, \mathrm{~B}$ ) $155 \mathrm{~cm}$ and C) $170 \mathrm{~cm}$, respectively. The experimentally observed transfer was normalized with respect to the $\mathrm{CP}$ signal at zero mixing time. The corresponding transient response of the probe is shown in the second row. The third row shows the effective field over one cycle of the C7 sequence calculated from the respective pulse shapes. DQ scaling factors $\boldsymbol{a}_{\mathbf{2}, \pm 2}^{(\mp \mathbf{2})}$ (green) and $\boldsymbol{a}_{2, \pm 2}^{(\mp 2, \mp 2)}$ (blue) are shown in the fourth row. The fifth and sixth row show the CS scaling factors $\left(\boldsymbol{a}_{\mathbf{1 , 0}}^{(\mathbf{0 , 0})}\right)$ and the magnitude of the CSA-CSA cross terms (calculated under the assumption of axially symmetric tensors and multiplied by the MAS frequency), respectively. The dashed red 
lines indicate the expected values if ideal rectangular pulses are considered. See text for further discussion.

\subsection{Asynchronous Recoupling}

In case of setup $\mathrm{C}$ (Figure 4, third column), the effective field of the $\mathrm{C} 7$ sequence does not vanish in the given range of rf fields and, thus, the resonance condition is never matched and no

significant polarization transfer is found. Nevertheless, the calculated coefficients $a_{2, \pm 2}^{(\mp 2, \mp 2)}$ at the generalized triple-mode resonance condition of Eq. (15) are found to be large. This indicates that an asynchronous implementation of the $\mathrm{C} 7$ sequence to match the triple-mode resonance condition, $\omega_{\mathrm{r}}+2 \omega_{\mathrm{m}}+2 \omega_{\text {eff }}=0$, should lead to efficient transfer. This can most easily be achieved by changing the MAS frequency. As the modulation frequency of the pulse sequence is not affected, this change in the MAS frequency directly compensates the additional effective field. Figure 5A shows a condition of efficient transfer for a spinning frequency of approximately $250 \mathrm{~Hz}$ less than the nominal value. This is found to be in good agreement with the calculated resonance condition (Figure 5B). Such an asynchronous implementation is possible in all cases were effective fields are present. Figure S2 shows the results for changes in the MAS frequency in case of setup A (cable length $125 \mathrm{~cm}$ ). As an alternative, also the amplitude or the length of one pulse of the C-element can be changed to re-establish dipolar recoupling by changing the effective field[30]. For the latter case, this is demonstrated in Figures S3 and S4.

It is obvious, that the conditions for good transfer in all these experiments strongly depend on the values of the effective field. As $\omega_{\text {eff }}$ is determined by the explicit shape of the transient the resonance condition varies strongly between the experimental setups. In order to obtain optimum transfer, a two-parameter grid search on the respective parameters is necessary. This can lead to impractically long optimization times, especially, if the sample has a low signal-to-noise ratio. 
Therefore, asynchronous implementations of C7 for the purpose of transient compensation are not a good solution for routine experiments.

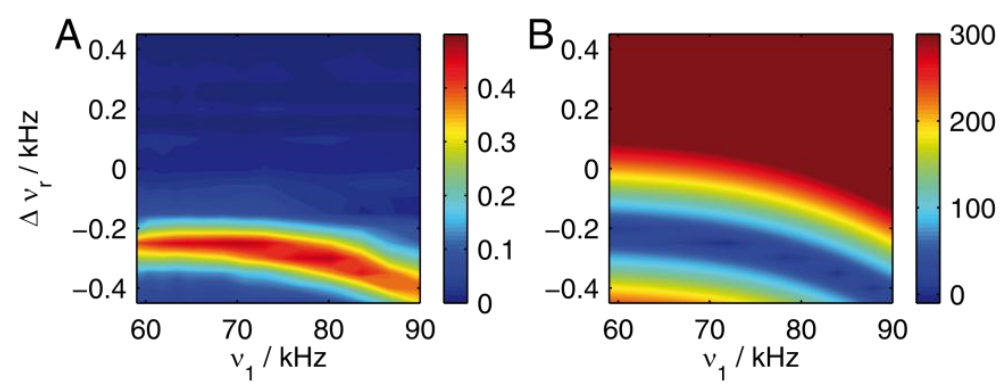

Figure 5: Asynchronous implementation of POST-C7 by changing the MAS frequency by $\boldsymbol{\Delta} \boldsymbol{v}_{\mathbf{r}}$. at an external field of 7.1 T. A) Experimental transfer as a function of the rf field $\boldsymbol{v}_{\mathbf{1}}$ and $\boldsymbol{\Delta} \boldsymbol{v}_{\mathbf{r}}$. B) Calculated values $\left|-\boldsymbol{v}_{\mathbf{r}}+\mathbf{2} \boldsymbol{v}_{\mathrm{m}}+\mathbf{2} \boldsymbol{v}_{\text {eff }}\right|$. The experimental condition for transfer is in good agreement with the calculated resonance condition. The experimental setup $\mathrm{C}$ with a cable length of $170 \mathrm{~cm}$ (Figure 4, third column) was used.

\subsection{The Transient-Adapted Basic Element}

An alternative way to minimize the effective field of the C7 sequence and to restore the original C7 resonance condition was proposed by Weber et al. [5] using a transient-adapted basic element where the phase of the first $90^{\circ}$ pulse of each POST element is shifted by an experimentally optimized amount $\zeta$ (Figure 6A). As both, the MAS frequency and the modulation frequency of the $\mathrm{C}$-sequence are unchanged, the calculated deviation from the resonance condition (Figure 6B) indicates, that the effective field vanishes due to a complex rotation over a symmetry cycle. However, the effective fields and, consequently, the optimal values of $\zeta$ and $v_{1}$ depend on the experimental setup (the results for a different setup are shown in Figure S5). Furthermore, a twoparameter optimization of rf-field amplitude and phase shift is necessary for optimum efficiency which makes this approach also cumbersome to use for practical applications. 


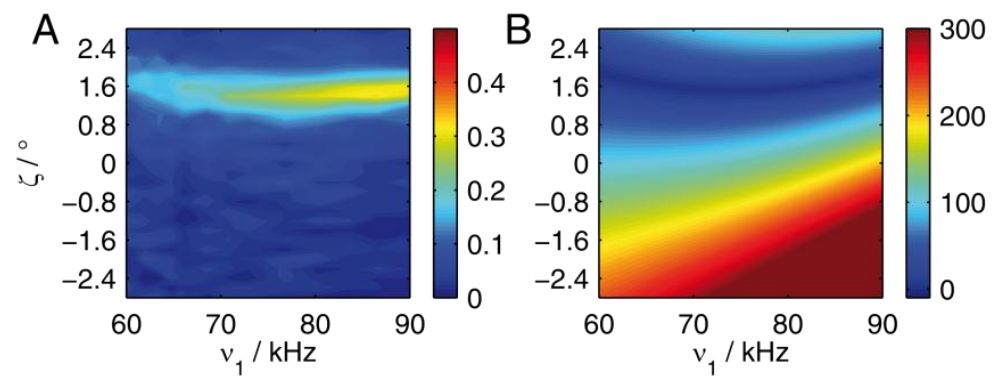

Figure 6: Transient-adapted POST-C7. A) experimental transfer as a function of the rf field $\boldsymbol{v}_{\mathbf{1}}$ and the phase shift $\zeta$ of the $90^{\circ}$ pulse of the POST pulse. B) Calculated values $\mid-\boldsymbol{v}_{\mathbf{r}}+\mathbf{2} \boldsymbol{v}_{\mathbf{m}}+$ $\mathbf{2} \boldsymbol{v}_{\text {eff }} \mid$. By changing the phase, the effective field is changed and becomes zero and the doublemode resonance condition is matched. Setup C (Figure 4 last column, cable length $170 \mathrm{~cm}$ ) was used at the $300 \mathrm{MHz}$ spectrometer.

\section{Implementation of Transient-Compensated Pulses as Basic Elements}

4.1 Modifications of the Basic ElementApplication of transient-compensated amplitudeshaped pulses inside symmetry-based sequences requires small modifications to the basic element for optimum experimental performance. In the following, these modifications will be discussed based on the Floquet description. The first-order effective Hamiltonian of the $\mathrm{C} 7 \frac{1}{2}$ sequence given by Eq. (16) contains the resonant double-quantum terms (Eq. (18)) as well as non-resonant error terms proportional to the chemical-shift scaling factors in Eq. (19). For good recoupling performance, the double-quantum scaling factor has to be high while the chemical-shift scaling factor should simultaneously be as low as possible. In order to quantify the efficiency of the recoupling sequence with the transient-compensated amplitude-shaped pulses as basic elements, both scaling factors are calculated as a function of the rise and fall time of the pulses and the maximum rf-field amplitude for an amplitude-shaped POST (aPOST) element (Figure 7A). In the limit of hard pulses (Figure 7A, $\tau_{\text {edge }}=0$ ) and using an ideal rf-field amplitude of $7 \omega_{\mathrm{r}}$, a pure DQ Hamiltonian is obtained as the chemical-shift scaling factor vanishes. At this condition, the effective flip angle of the C-element is zero. However, it can easily be seen from Figure 7A that the double-quantum scaling factors rapidly decrease to zero with increasing rise and fall time of 
the aPOST element. Due to the shaped amplitudes and the different length of the three pulses contained in the POST element, the net rotation over the $\mathrm{C}$ element is no longer zero. Therefore, the C-element does not correspond to a unity propagator and the effective field $\omega_{\text {eff }}$ is no longer zero. Hence, the resonance condition of the synchronous C7 sequence is not matched anymore. Compared to the hard-pulse limit $\left(\tau_{\text {edge }}=0\right)$, the condition of optimum chemical-shift compensation also narrows down considerably (Figure 7A) when finite edge times are used. In principle, there are two approaches to re-establish the resonance conditions of $\mathrm{C} 7{ }_{2}^{1}$. All sequences which have a non-vanishing effective field can be implemented asynchronously to match the three-frequency resonance condition of Eq. (15) using the concepts developed in section 3.3. However, for experimentally feasible transition times the calculated DQ scaling factors start decreasing (see Figure S6). Therefore, we will not pursue this alternative further and focus on a synchronous implementation in the following.

In order to restore efficient double-quantum recoupling under rotor-synchronized rf irradiation, the basic $\mathrm{C}$ element has to be modified such that it corresponds again to a unity propagator without effective field. The simplest way to achieve this is to scale the rf-field amplitudes such that the flip-angles of the individual amplitude-shaped pulses are equal to the theoretical values (Figure 7B). The resulting amplitude-shaped flip-angle corrected POST element (afc1 $\overline{4} 3$ ) can be used in a rotor-synchronized protocol. The calculated scaling factors as a function of edge time and rf-field amplitude are shown in Figure 7B. High double-quantum scaling factors are obtained over the whole range of parameters. Especially for longer transition times, however, the chemical-shift compensation depends strongly on the rf-field amplitudes and is no longer perfect (Figure 7B). Due to the different rf-field amplitudes, the internal symmetry of the basic element is broken [27] and an incomplete averaging of the chemical shift is obtained during a single C element. By splitting the central $2 \pi$ pulse of the aPOST-element into a $\pi / 2$ and a $3 \pi / 2$ pulse, the 
symmetry of the nutation trajectory can be restored. The modified POST element $(\mathrm{a} \pi / 2)_{\varphi}(\mathrm{a} 3 \pi / 2)_{\varphi+\pi}(\mathrm{a} \pi / 2)_{\varphi+\pi}(\mathrm{a} 3 \pi / 2)_{\varphi}(\mathrm{a} 1 \overline{3} \overline{1} 3)$ corresponds to a unity propagator and suppresses chemical shifts well in first-order approximation (Figure 7C). Further improvement can be obtained by the flip-angle corrected TIPTOP [23] element (amplitude-shaped flip-angle corrected afc $1 \overline{3} \overline{1} 3$ pulse, Figure 7D) which does not only provide chemical-shift compensation but also high double-quantum scaling factors even for longer pulse rise times. It is evident from the third column in Figure 7D, that the condition for good chemical-shift compensation is as broad as in the case of ideal rectangular pulses. A very similar result can be obtained for the amplitudeshaped flip-angle corrected afc $1 \overline{1111} 111$ basic element, where all the three pulses of the original POST pulse are built up from discrete amplitude-shaped $\pi / 2$ pulses (Figure 7E). This pulse has the advantage that the transverse components of the chemical shift in the interaction frame $\left(\left|a_{1, \pm 1}^{(0,0)}\right|\right)$ are averaged to zero already over one basic C element while they are only compensated over an entire C7 sequence for the TIPTOP (afc1 $\overline{3} \overline{1} 3$ ) element (see also Figure S7 and S8 for a comparison of different basic elements). The last two basic elements, TIPTOP (afc1 $\overline{3} \overline{1} 3$ ) and afc1 $\overline{1111} 111$ are the most promising candidates for an experimental implementation. It turned out during the course of experimental optimizations that the TIPTOP pulse is slightly more robust with respect to $\mathrm{rf}$ mismatch and chemical-shift offsets than the afc11111111. The reason why this basic element performs worse experimentally might be attributed to a higher loss of magnetization during the increasing time, where the rf field is zero. Another reason might be that small imperfections of the compensation occur strongest at the conjunctions of the pulses and thus their effects accumulate more for the afc1 $\overline{1111} 111$. 

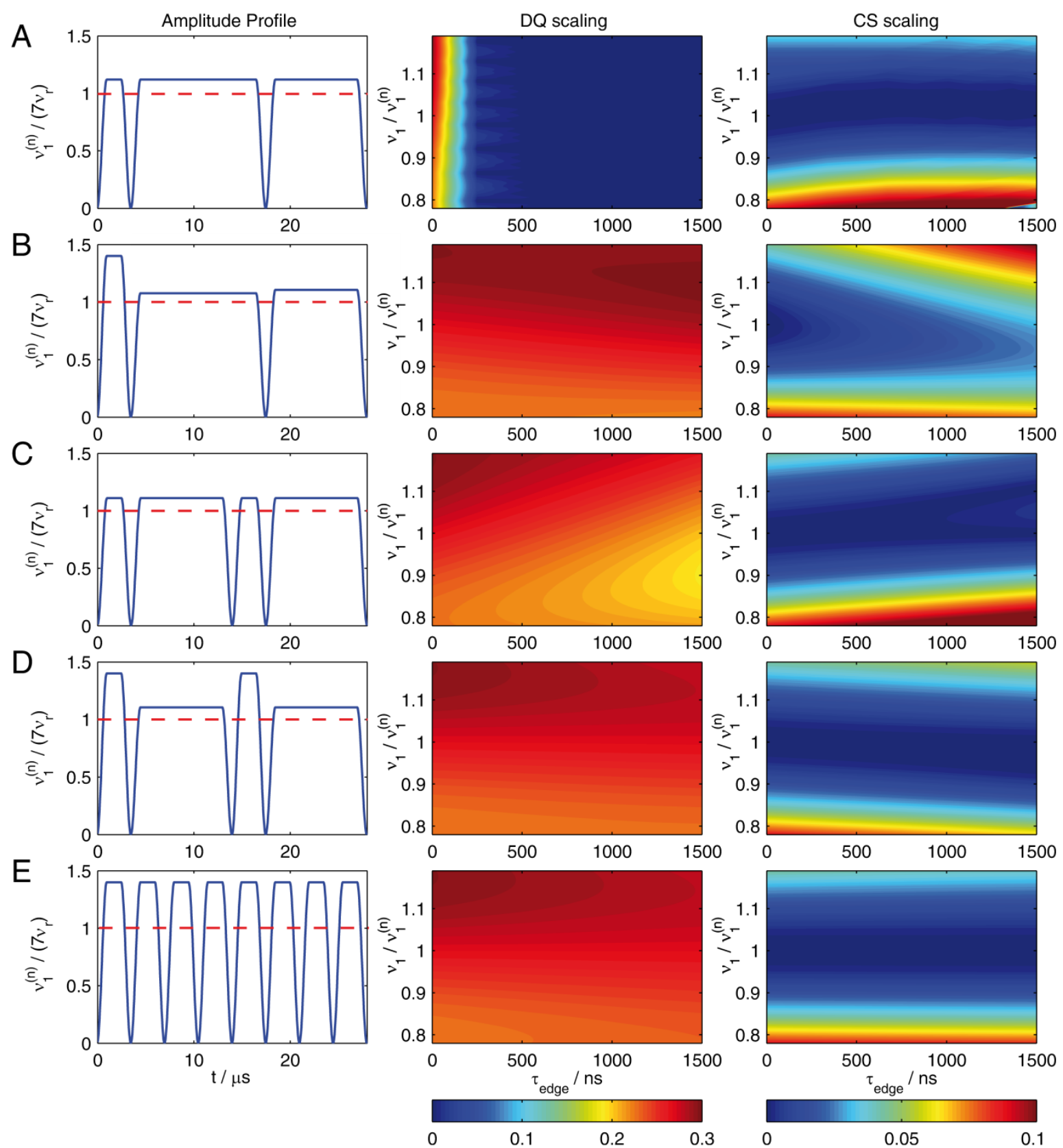

Figure 7: Magnitude of DQ ( $2^{\text {nd }}$ column) and CS scaling factors ( $3^{\text {rd }}$ column) for five different amplitude-shaped composite pulses derived from the POST pulse used as basic C-element in rotor-synchronized $\mathrm{C7}_{\mathbf{2}}^{\mathbf{1}}$ protocol as a function of edge times and applied rf-fields. A) amplitudeshaped POST B) amplitude-shaped POST with flip-angle correction C) amplitude-shaped $\mathbf{1 3} \overline{1} 3$ D) amplitude-shaped $\mathbf{1} \overline{\mathbf{3}} \mathbf{3}$ with flip-angle correction. E) amplitude-shaped $\mathbf{1 1 1 1 1 1 1 1}$ pulse with flip-angle correction. The MAS frequency was set to $10.204 \mathrm{kHz}$ corresponding to a length of $28 \mu \mathrm{s}$ for the rotor synchronized POST element. The data in subfigures A,B and D are the same as in reference [32] and are shown here for easy comparison. 


\subsection{Improved Robustness against Variations in the Experimental Setup}

The major advantage of using transient-compensated basic elements is, that the rf field acting on the spins can be controlled to a high extent by the spectrometer operator. Adjusting the compensated input shapes to the current experimental conditions prior to the experiment will guarantee that a well-defined rf field acts on the nuclear spins. In comparison to a re-optimization of the whole pulse sequence which can involve a time-consuming multi-parameter grid search (see Sections 3.3 and 3.4), the determination of the compensated input shapes only takes a few minutes as it does not require an observable NMR signal from the sample. A comparison between the experimental performance of conventional hard-pulse POST-C7 and transient-compensated TIPTOP-C7 DQ recoupling at $10.2 \mathrm{kHz}$ MAS and an external field of 7.1 Tesla is shown in Figure 8A-D. Not only the rf fields for optimal transfer (Figure 8A) show a strong dependence on the cable length, also the corresponding build-up curves at the optimized rf fields have a large spread from good agreement with the simulated data under ideal conditions to unreasonable behavior (Figure 8C). In contrast, the time dependence of the polarization transfer for transientcompensated sequence is in very good agreement with the numerical simulations and the variation between the experiments is reduced to a minimum allowing an accurate determination of the internuclear distance (Figure 8D). Furthermore, the rf field of optimal transfer is independent of the cable length. We have already shown [23], that the same behavior is obtained on a different spectrometer at an external magnetic field of 9.4 Tesla. For a better comparability, build-up curves and matching profiles from reference [23] are shown again in Figure 8E-H. Figure 8I-L illustrates the recoupling behavior of POST-C7 and TIPTOP-C7 on the $300 \mathrm{MHz}$ spectrometer but at an increased a MAS frequency of $14.3 \mathrm{kHz}$. Due to the rotor-synchronization of the pulse sequence, the pulse lengths decrease by about $30 \%$. As the time-constant of the pulse transients is unchanged, a higher fraction of each pulse contains deviations from the intended 
amplitudes and phases. Consequently, the effective field increases. Figure 8I and $\mathrm{K}$ show, that this significantly degrades the efficiency of the conventional POST-C7 experiment. The efficiency of the compensated TIPTOP-C7, however, is unaffected by these changes (Figure $8 \mathrm{~J} / \mathrm{L}$ ). These findings underline that there is no universally "good" or "bad" cable, which should be used or avoided. Even if there are experimental conditions, where (close to) optimal performance of a given sequence can simply be achieved by selection of a proper cable length, the effective field originating from the specific shape of the pulse transients is also influenced by several other parameters. For a given symmetry $N, n, v$ changing the MAS frequency leads to a changed pulse length and thus to different effective fields.
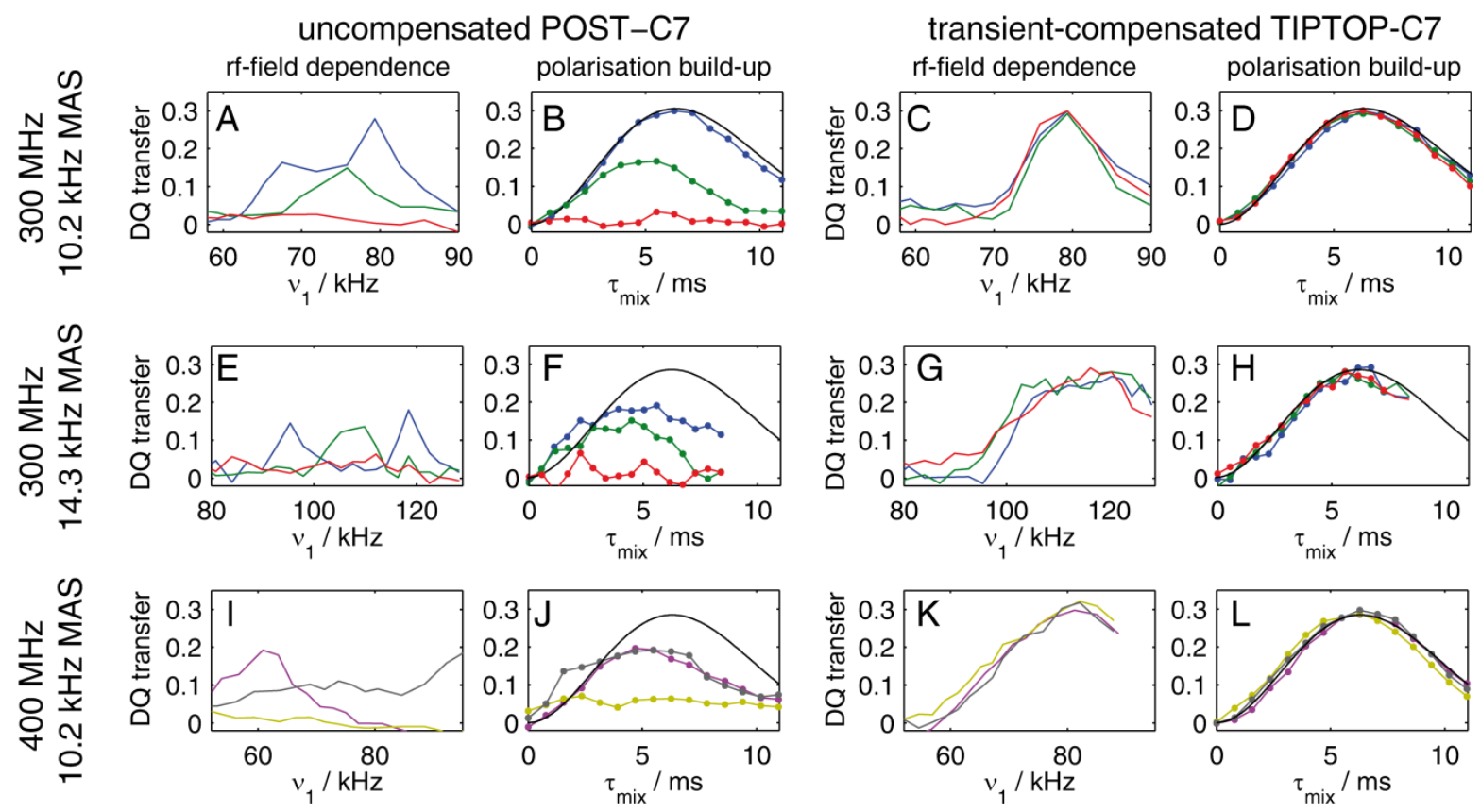

Figure 8: Improved robustness of POST-C7 DQ recoupling with respect to differences in the experimental setup. Matching profile of A/E/I) uncompensated POST-C7 and B/F/J) the compensated TIPTOP-C7 sequence for three different cables and the corresponding build-up curves at the rf field of optimum transfer for $\mathrm{C} / \mathrm{G} / \mathrm{K}$ ) the uncompensated POST and D/H/L) the compensated TIPTOP basic element. The data shown in subfigures A-D and I-L was recorded at the $300 \mathrm{MHz}$ spectrometer and an MAS frequency of 10.2 and $14.3 \mathrm{kHz}$, respectively. Matching profiles and build-up curves from E-H, were recorded at $10.2 \mathrm{kHz}$ MAS and an external magnetic field of $9.4 \mathrm{~T}$. The colors indicate different experimental setups, the black line represents simulated polarization transfer. 


\subsection{Application to $\mathrm{R}_{2}^{6}$ double-quantum recoupling}
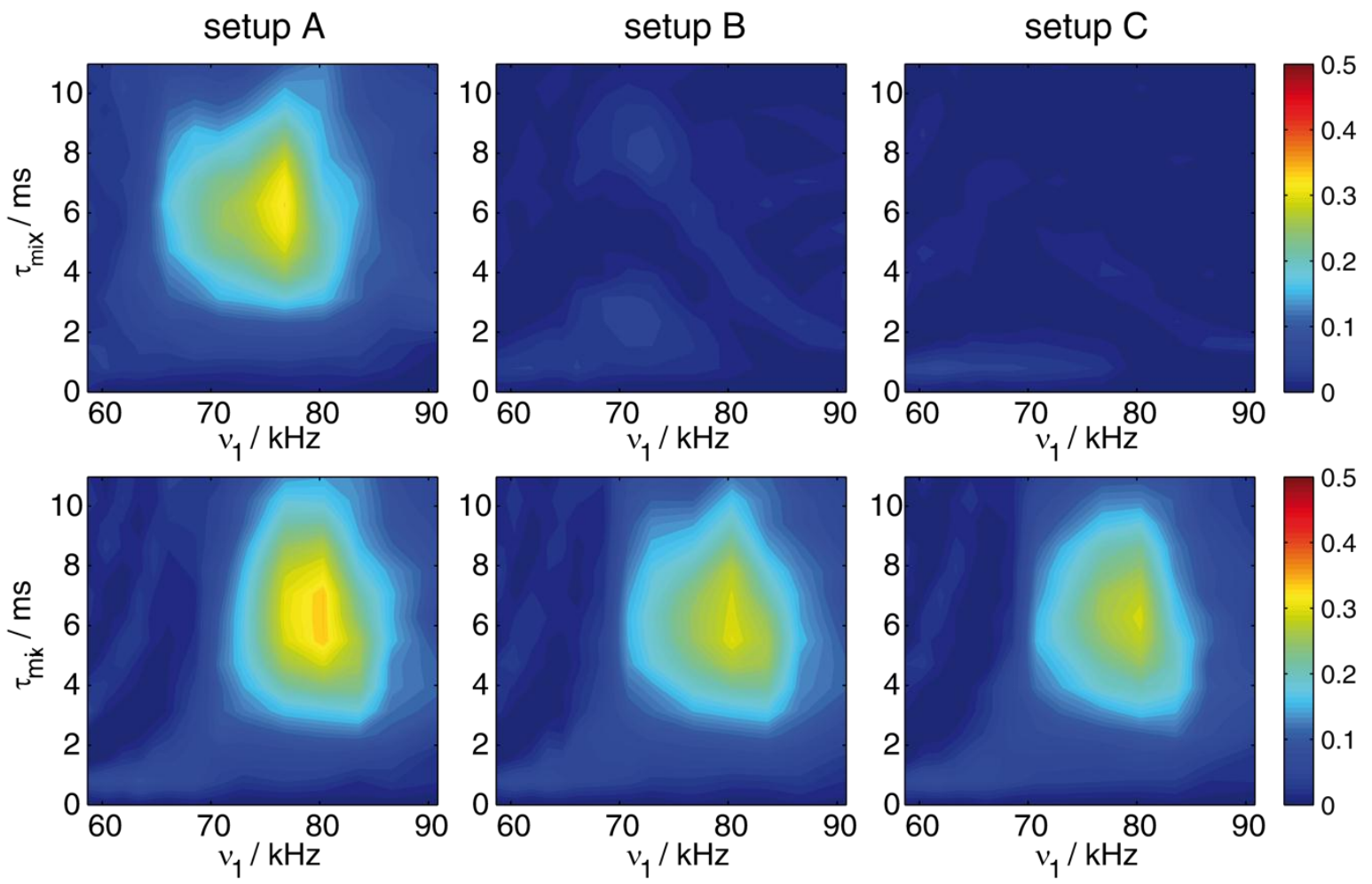

Figure 9: Experimental R14 ${ }_{2}^{6}$ double-quantum transfer in di-ammonium phthalate at $10.2 \mathrm{kHz}$ MAS. The external magnetic field was $7.1 \mathrm{~T}$, a $(\pi / 2)_{\varphi}(3 \pi / 2)_{\varphi+\pi}$ basic element was used. The top and the bottom row show the results for conventional hard pulses and transient-compensated amplitude-shaped pulses with flip-angle correction, respectively. The results are shown for the same three different cables A, B and C as used in Fig. 8.

The concepts for the application of transient-compensated pulses in symmetry-based Csequences, which have been illustrated for the example of POST-C7 double-quantum recoupling, can easily be extended to R-sequences. Here, the commonly-used basic element $(\pi / 2)_{\varphi}(3 \pi / 2)_{\varphi+\pi}$ is substituted by an amplitude-shaped and flip-angle corrected $(\pi / 2)_{\varphi}(3 \pi / 2)_{\varphi+\pi}$ pulse. As an example, we use the double-quantum recoupling sequence $\mathrm{R} 14_{2}^{6}$, which has the same rf-requirements as POST-C7 $\left(\omega_{1}=7 \omega_{\mathrm{r}}\right)$ [6]. Using the same three experimental setups as before, we measured the double-quantum polarization transfer in di-ammonium phthalate as a function of rf field and mixing time at a MAS frequency of $10.2 \mathrm{kHz}$ MAS and a static magnetic field of $7.1 \mathrm{~T}$. The 
results are given in Figure 9. Using conventional hard pulses (first row), it can easily be seen that in two of the three cases no transfer is observed experimentally. This indicates that for a given experimental setup also a change in the symmetry of the pulse sequence can cause strong variations of the observed results. Using transient-compensated pulses (second row of Figure 9) however, the influence of the experimental setup can again be minimized to a high extent leading to reproducibly high efficiencies.

\section{$5 \quad$ Materials and Methods}

$1,2-{ }^{13} \mathrm{C}_{2}$-glycine was purchased from Cambridge Isotopes and used without further purification. Phthalic acid-a,a- ${ }^{13} \mathrm{C}_{2}$ was purchased from Isotec. A mixture with unlabeled phthalic acid in a molar ratio of 1:7 was prepared in order to avoid intermolecular contacts. The di-ammonium salt, which has a shorter $T_{1}$ relaxation time, was synthesized by adding an excess of aqueous ammonia and crystallized by lyophilization.

All experiments on di-ammonium phthalate at an external field of 7.1 Tesla were carried out on a Bruker Avance III HD spectrometer using a Bruker $1.9 \mathrm{~mm}$ double-resonance probe. The corresponding ${ }^{13} \mathrm{C}$ Larmor frequency was $75.5 \mathrm{MHz}$. Experiments at $400 \mathrm{MHz}$ were performed as described in reference [23]. Both spectrometers are equipped with boards for extended pulseshape memory. Before carrying out the experiments, the probe was tuned to a minimum of the reflected power on both, proton and carbon channel. In order to quantify the double-quantum efficiency, the DQ transfer between the two resonances of di-ammonium phthalate was measured as a function of the mixing time for a series of rf fields. The experimentally measured intensities were normalized to the intensity of the selectively excited signal at zero mixing time. Phase and amplitude of the $B_{1}$ field in the coil were measured using a pick-up coil placed in close proximity to the NMR coil (inductive coupling $-40 \mathrm{~dB}$ ). For further information on this modification, the 
reader is referred to the Figure $S 9$ of the supporting information. Digitization of the induced current was performed on a Tektronix TDS7104 oscilloscope with a sampling rate of 5 Gsamples/s. After digital quadrature demodulation of the signal and digital filtering, the pulse envelopes were obtained with a time resolution of $50 \mathrm{~ns}$. The compensated pulse shapes were calculated (see Section 2.1) and converted into a Bruker shape file using the highest possible time resolution (50 ns). The digital signal processing was performed in MATLAB [33].

Numerical simulations were performed using the GAMMA spin-simulation environment [34]. A set of $538 \mathrm{ZCW}$ crystallite orientations [35] was used for powder averaging. The MAS frequency was set to $10.204 \mathrm{kHz}$ for all simulations and Floquet calculations. All experiments were performed at $10.2 \mathrm{kHz}$. Orientations of dipole-dipole and chemical-shift tensors were taken from quantum-chemical calculations based on the crystal structure [36]. High-power continuous-wave (cw) proton decoupling $\left(v_{1}=155 \mathrm{kHz}\right)$ was applied during the recoupling period.

The interaction-frame transformation required for the Floquet calculations was calculated on the basis of Eq. (11). The integral was evaluated numerically as

$$
\widehat{U}(t)=\prod_{k} \exp \left(-i \mathcal{H}_{\mathrm{rf}}(k \delta t) \delta t\right)
$$

and the time-dependent amplitudes $\omega_{1}(k \delta t)$ and phases $\phi(k \delta t)$ (see also Eq. (10)) were used from a discrete experimental dataset with a time resolution of $\delta \tau=50 \mathrm{~ns}$. The corresponding propagator $U_{0}$ of a single $\mathrm{C}$ element is then calculated numerically assuming that the rf field is constant on the timescale of $\delta \tau$. Using this propagator, the propagators of the remaining $\mathrm{C}$ elements can be calculated by rotation of $U_{0}$ around the z-axis in integer multiples of the characteristic phase increment of the pulse-sequence symmetry. By explicit calculation of the expectation values of the $\mathrm{x}, \mathrm{y}$ and $\mathrm{z}$-operators, the interaction-frame trajectory is calculated over $1200 \mathrm{CN}$-cycles in order to provide sufficient frequency resolution. The relevant Cartesian 
Fourier coefficients describing the interaction-frame transformation of these operators can be calculated using a manual Fourier transformation with the basic frequencies $\omega_{\mathrm{m}}$ and $\omega_{\text {eff }}$. The latter as well as the corresponding effective rotation axis are determined by analyzing the first $\mathrm{CN}$-cycle using the quaternion formalism [37]. In a final step, the coefficients are converted from a Cartesian to a spherical tensor representation.

\section{Conclusion and Outlook}

In this publication, we demonstrated that a triple-mode Floquet description of symmetry-based recoupling allows the quantification of the effects of real pulses on the performance of the pulse sequence. Due to the pulse transients, the flip angles of the individual pulses deviate from the intended values causing a non-vanishing effective field over a symmetry cycle. Experimentally, transfer is only observed, if the rf field of the sequence can be adjusted such that the effective field becomes zero. Otherwise, this additional modulation leads to a shift of the resonance condition and reduced or no recoupling is observed. Alternatively, the application of a transientadapted basic element or an asynchronous implementation to match the three frequency resonance condition is possible. However, in all these cases the exact location of the resonance conditions exhibit a strong dependence on the details of the experimental setup which determine the magnitude of the effective field.

These deviations can be minimized to a large extent using transient-compensated pulses as basic elements of the symmetry-based sequences. Such pulses can be calculated using linear-response theory. As the rf field acting on the spins is under full control of the experimentalist, the small modifications to the basic elements of symmetry-based sequences which are necessary to take into account for the finite rising and falling times of the resulting amplitude-shaped pulses can 
easily be implemented. They retain the nominal flip-angles and the internal symmetry of the basic elements leading to reproducibly high transfer efficiencies and a strongly improved robustness against differences in the experimental conditions.

\section{Acknowledgement}

The authors want to thank David Brunner, Andrin Doll, Johannes Hellwagner, and Kong Ooi Tan for stimulating discussions. Alexander Daepp is acknowledged for the technical support. This work was supported by the Swiss National Science Foundation (Grant 200020 134681)

\section{References}

[1] M. Mehring, J.S. Waugh, Phase transients in pulsed NMR spectrometers, Rev. Sci. Instrum. 43 (1972) 649-653. doi:10.1063/1.1685714.

[2] J.D. Ellett, M.G. Gibby, U. Haeberlen, L.M. Huber, M. Mehring, A. Pines, et al., Spectrometers for Multiple-Pulse NMR, Adv. Magn. Reson. 662 (1971) 117-176. doi:10.1016/B978-0-12-025505-4.50009-0.

[3] T.M. Barbara, J.F. Martin, J.G. Wurl, Phase transients in NMR probe circuits, J. Magn. Reson. 93 (1991) 497-508. doi:10.1016/0022-2364(91)90078-8.

[4] A.J. Vega, Controlling the effects of pulse transients and RF inhomogeneity in phasemodulated multiple-pulse sequences for homonuclear decoupling in solid-state proton NMR., J. Magn. Reson. 170 (2004) 22-41. doi:10.1016/j.jmr.2004.05.017.

[5] J. Weber, M. Seemann, J. Schmedt auf der Günne, Pulse-transient adapted C-symmetry pulse sequences., Solid State Nucl. Magn. Reson. 43-44 (2012) 42-50. doi:10.1016/j.ssnmr.2012.02.009.

[6] M. Carravetta, M. Edén, O.G. Johannessen, H. Luthman, P.J.E. Verdegem, J. Lugtenburg, et al., Estimation of Carbon-Carbon Bond Lengths and Medium-Range Internuclear Distances by Solid-State Nuclear Magnetic Resonance, J. Am. Chem. Soc. 123 (2001) 10628-10638. doi:10.1021/ja016027f. 
[7] U. HAEBERLEN, Introduction, Academic Press Inc, 1976. doi:10.1016/B978-0-12025561-0.50007-1.

[8] M. Mehring, Principles of High Resolution NMR in Solids, 2nd ed., Springer-Verlag, Berlin, Heidelberg, New York, 1983. doi:10.1007/978-3-642-68756-3.

[9] R.W. Vaughan, A Simple, Low Power, Multiple Pulse NMR Spectrometer, Rev. Sci. Instrum. 43 (1972) 1356. doi:10.1063/1.1685924.

[10] W.-K. Rhim, D.D. Elleman, R.W. Vaughan, Analysis of multiple pulse NMR in solids, J. Chem. Phys. 59 (1973) 3740-3749. doi:10.1063/1.1680545.

[11] W.-K. Rhim, D.D. Elleman, R.W. Vaughan, Analysis of multiple pulse NMR in solids, J. Chem. Phys. 59 (1973) 3740-3749. doi:10.1063/1.1680545.

[12] D.P. Burum, M. Under, R.R. Ernst, A new "tune-up" NMR pulse cycle for minimizing and characterizing phase transients, J. Magn. Reson. 43 (1981) 463-471. doi:10.1016/00222364(81)90058-5.

[13] E. Vinogradov, P. Madhu, S. Vega, High-resolution proton solid-state NMR spectroscopy by phase-modulated Lee-Goldburg experiment, Chem. Phys. Lett. 314 (1999) 443-450. doi:10.1016/S0009-2614(99)01174-4.

[14] M.H. Levitt, Symmetry in the design of NMR multiple-pulse sequences, J. Chem. Phys. 128 (2008) 52205. doi:10.1063/1.2831927.

[15] M.H. Levitt, Symmetry-Based Pulse Sequences in Magic-Angle Spinning Solid-State NMR, in: Encycl. Magn. Reson., 2007: pp. 165-196. doi:10.1002/9780470034590.emrstm0551.

[16] M. Hohwy, H.J. Jakobsen, M. Edén, M.H. Levitt, N.C. Nielsen, M. Eden, Broadband dipolar recoupling in the nuclear magnetic resonance of rotating solids: A compensated C7 pulse sequence, J. Chem. Phys. 108 (1998) 2686. doi:10.1063/1.475661.

[17] K. Takeda, Chapter 7 - Highly Customized NMR Systems Using an Open-Resource, Home-Built Spectrometer, in: Annu. Reports NMR Spectrosc., 2011: pp. 355-393. doi:10.1016/B978-0-08-097072-1.00007-8.

[18] K. Takeda, Y. Tabuchi, M. Negoro, M. Kitagawa, Active compensation of rf-pulse transients, J. Magn. Reson. 197 (2009) 242-244. doi:10.1016/j.jmr.2008.12.012.

[19] Y. Tabuchi, M. Negoro, K. Takeda, M. Kitagawa, Total compensation of pulse transients inside a resonator, J. Magn. Reson. 204 (2010) 327-332. doi:10.1016/j.jmr.2010.03.014.

[20] P.E. Spindler, Y. Zhang, B. Endeward, N. Gershernzon, T.E. Skinner, S.J. Glaser, et al., Shaped optimal control pulses for increased excitation bandwidth in EPR., J. Magn. Reson. 218 (2012) 49-58. doi:10.1016/j.jmr.2012.02.013. 
[21] T.W. Borneman, D.G. Cory, Bandwidth-limited control and ringdown suppression in highQ resonators., J. Magn. Reson. 225 (2012) 120-9. doi:10.1016/j.jmr.2012.10.011.

[22] T. Kaufmann, T.J. Keller, J.M. Franck, R.P. Barnes, S.J. Glaser, J.M. Martinis, et al., DAC-board based X-band EPR spectrometer with arbitrary waveform control., J. Magn. Reson. 235 (2013) 95-108. doi:10.1016/j.jmr.2013.07.015.

[23] J.J. Wittmann, K. Takeda, B.H. Meier, M. Ernst, Compensating Pulse Imperfections in Solid-State NMR Spectroscopy: A Key to Better Reproducibility and Performance, Angew. Chemie Int. Ed. 54 (2015) 12592-12596. doi:10.1002/anie.201504782.

[24] D. Havelock, S. Kuwano, M. Vorländer, eds., Handbook of Signal Processing in Acoustics, Springer New York, New York, NY, 2008. doi:10.1007/978-0-387-30441-0.

[25] D.D. Rife, J. Vanderkooy, Transfer-Function Measurement with Maximum-Length Sequences, J. Audio Eng. Soc. 37 (1989) 419-444.

[26] P. Bloomfield, Fourier Analysis of Time Series: An Introduction, 2nd ed., WileyInterscience, New York, NY, 2013.

[27] F.-C. Chou, H.-K. Lee, J.C.C. Chan, Internal symmetry of basic elements in symmetrybased recoupling sequences under magic-angle spinning., J. Chem. Phys. 133 (2010) 114503. doi:10.1063/1.3469766.

[28] I. Scholz, J.D. van Beek, M. Ernst, Operator-based Floquet theory in solid-state NMR, Solid State Nucl. Magn. Reson. 37 (2010) 39-59. doi:10.1016/j.ssnmr.2010.04.003.

[29] E. Vinogradov, P.K. Madhu, S. Vega, Strategies for High-Resolution Proton Spectroscopy in Solid-State NMR, in: Top. Curr. Chem., 2005: pp. 33-90. doi:10.1007/b98648.

[30] K.O. Tan, M. Rajeswari, P.K. Madhu, M. Ernst, Asynchronous symmetry-based sequences for homonuclear dipolar recoupling in solid-state nuclear magnetic resonance, J. Chem. Phys. 142 (2015) 065101. doi:10.1063/1.4907275.

[31] F.J. Dyson, The radiation theories of Tomonaga, Schwinger, and Feynman, Phys. Rev. 75 (1949) 486-502. doi:10.1103/PhysRev.75.486.

[32] J.J. Wittmann, L. Hendriks, B.H. Meier, M. Ernst, Controlling spin diffusion by tailored rfirradiation schemes, Chem. Phys. Lett. 608 (2014) 60-67. doi:10.1016/j.cplett.2014.05.057.

[33] MATLAB The MathWorks Inc. Natick Massachusetts, version 7.14.0, (2012).

[34] S.A. Smith, T.O. Levante, B.H. Meier, R.R. Ernst, Computer Simulations in Magnetic Resonance. An Object Oriented Programming Approach, J. Magn. Reson. 106 (1994) 75105. doi:http://dx.doi.org/10.1006/jmra.1994.1008. 
[35] V.B. Cheng, H.H. Suzukawa, M. Wolfsberg, Investigations of a nonrandom numerical method for multidimensional integration, J. Chem. Phys. 59 (1973) 3992. doi:10.1063/1.1680590.

[36] R.A. Smith, The crystal structures of a series of salts of phthalic acid. Diammonium phthalate (NH4)2(OOC.C6H4.COO), Acta Crystallogr. Sect. B Struct. Crystallogr. Cryst. Chem. 31 (1975) 1773-1775. doi:10.1107/S0567740875006139.

[37] B. Blumich, H.W. Spiess, Quaternions as a Practical Tool for the Evaluation of Composite Rotations, J. Magn. Reson. 362 (1985) 356-362. doi:10.1016/0022-2364(85)90091-5. 


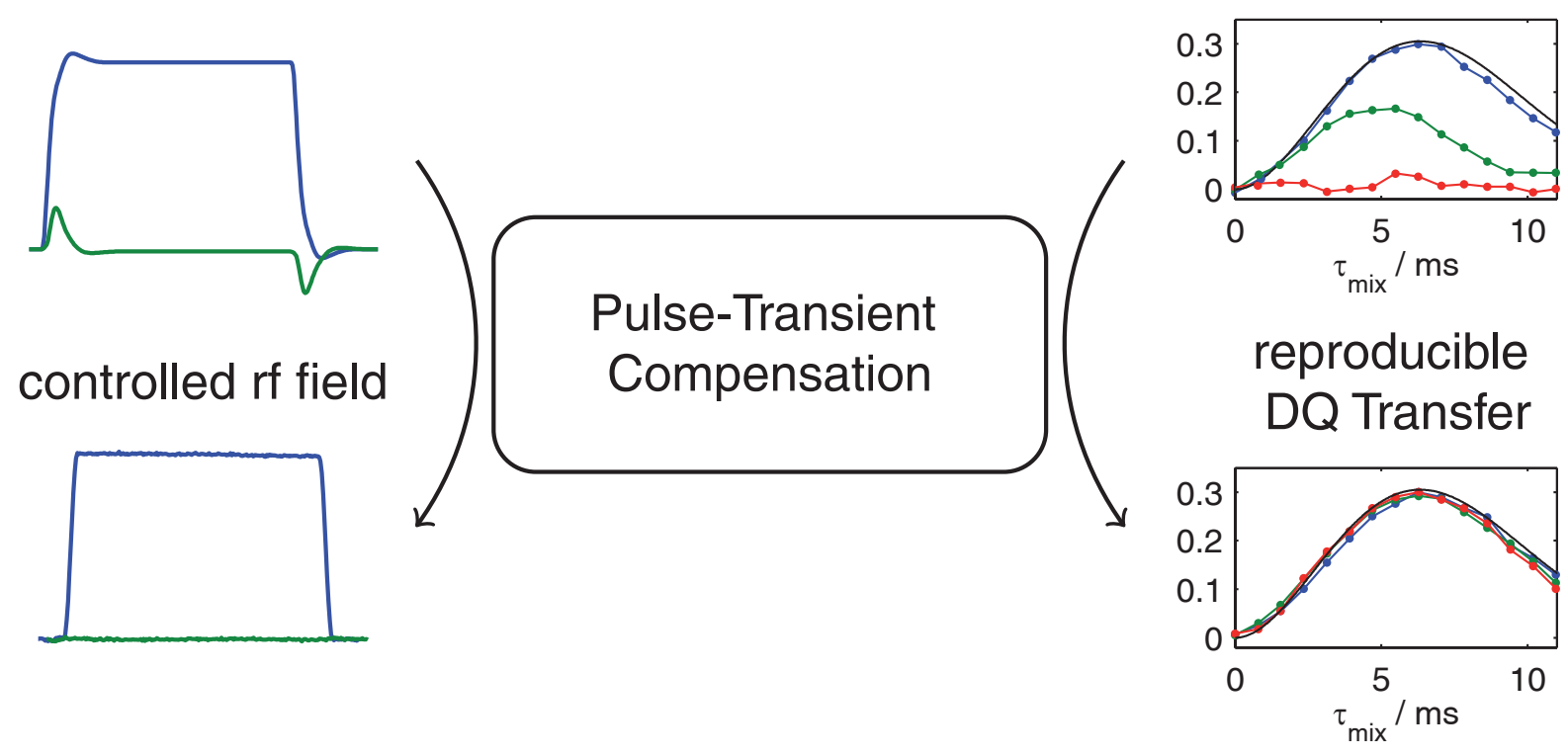

